\title{
On the moduli space of $\tau$-connections on a compact Riemann surface
}

\author{
Indranil Biswas
}

\begin{abstract}
Fix a $\tau$-connection $D_{L}$ on a line bundle $L$ over $X \times \mathbb{C}$, where $X$ is a compact connected Riemann surface of genus at least three. Let $\mathcal{M}_{X}\left(D_{L}\right)$ denote the moduli space of all semistable $\tau$-connections of rank $n$, where $n \geqslant 2$, with the property that the induced $\tau$-connection on the top exterior product is isomorphic to $\left(L, D_{L}\right)$. Let $\mathcal{M}_{Y}\left(D_{M}\right)$ be the similar moduli space for another Riemann surface $Y$ with $\operatorname{genus}(Y)=\operatorname{genus}(X)$, where $D_{M}$ is a $\tau$-connection on a line bundle $M$ over $Y \times \mathbb{C}$. We prove that if the variety $\mathcal{M}_{X}\left(D_{L}\right)$ is isomorphic to $\mathcal{M}_{Y}\left(D_{M}\right)$, then $X$ is isomorphic to $Y$.

Let $\mathcal{M}_{X}^{D}$ denote the moduli space of all rank $n$ flat connections on $X$. We prove that $\mathcal{M}_{X}^{D}$ determines $X$ up to finitely many Riemann surfaces. For the very general Riemann surface $X$, the variety $\mathcal{M}_{X}^{D}$ determines $X$.
\end{abstract}

\section{Introduction}

Let $X$ be a compact connected Riemann surface of genus $g$ which is at least three. The holomorphic cotangent bundle of $X$ will be denoted by $K_{X}$. Let $p$ (respectively, $\tau$ ) denote the projection of $X \times \mathbb{C}$ to $X$ (respectively, $\mathbb{C}$ ).

Let $E$ be an algebraic vector bundle of rank $n$ on $X \times \mathbb{C}$. We recall from [Sim94a, p. 87] that a $\tau$-connection on $E$ is an algebraic relative differential operator

$$
D: E \longrightarrow p^{*} K_{X} \otimes E
$$

satisfying the identity

$$
D(f s)=f D(s)+\tau d_{X} f \otimes s,
$$

where $s$ is a (locally defined) section of $E$ and $f$ is a (locally defined) function on $X \times \mathbb{C}$, and $d_{X}$ is the differential along $X$; the function $\tau$ on $X \times \mathbb{C}$ is defined above. This identity is equivalent to the condition that the relative differential operator $D$ is of order one and the symbol of the restriction of $D$ to $X \times\{\lambda\}$, where $\lambda \in \mathbb{C}$, is $\lambda$ times the identity automorphism of $\left.E\right|_{X \times\{\lambda\}}$. Therefore, for $\lambda \neq 0$, the operator $D / \lambda$ on $X \times\{\lambda\}$ is a holomorphic connection on $\left.E\right|_{X \times\{\lambda\}}$, and $D$ on $X \times\{0\}$ is a Higgs field on $\left.E\right|_{X \times\{0\}}$. Note that since $\operatorname{dim}_{\mathbb{C}} X=1$, the integrability condition $D \circ D=0$ is automatically satisfied.

If $V$ is a holomorphic vector bundle over $X$ equipped with a holomorphic connection $\nabla$, then degree $(V)=0$. Therefore, for any subbundle $F$ of $V$ preserved by $\nabla$ we have $\operatorname{degree}(F) / \operatorname{rank}(F)=$ $0=\operatorname{degree}(V) / \operatorname{rank}(V)$. In other words, $\nabla$ is semistable (see [Sim94a, p. 92] for the definition of semistability of an integrable system). A $\tau$-connection $D$ on $E$ is called semistable if for each $\lambda \in \mathbb{C}$, the restriction $\left.E\right|_{X \times\{\lambda\}}$ equipped with $\left.D\right|_{X \times\{\lambda\}}$ is semistable [Sim94a, p. 92]. Since any flat connection is semistable, $D$ is semistable if and only if the Higgs bundle defined by the Higgs field $\left.D\right|_{X \times\{0\}}$ on $\left.E\right|_{X \times\{0\}}$ is semistable.

Received 29 January 2003, accepted in final form 16 March 2003.

2000 Mathematics Subject Classification 14D20, $14 \mathrm{C} 34$.

Keywords: flat connection, Higgs bundle, moduli space, Torelli theorem.

This journal is (C) Foundation Compositio Mathematica 2004. 


\section{BISWAS}

In [Sim94a], Simpson constructed the moduli space of semistable $\tau$-connections (see [Sim94a, p. 104, Theorem 4.7]). Let $\mathcal{M}_{X}(n)$ denote the moduli space of all rank $n$ semistable $\tau$-connections.

If $D$ is a $\tau$-connection on $E$, then the line bundle $\bigwedge^{n} E$ has a $\tau$-connection induced by $D$. Let $L$ be a holomorphic line bundle over $X$ of degree zero. Fix a $\tau$-connection $D_{L}$ on $p^{*} L$. For example, if $\nabla$ is a unitary connection on $L$, then $\lambda \nabla$ over $X \times\{\lambda\}$, where $\lambda \neq 0$, and the Higgs bundle $(L, 0)$ over $X \times\{0\}$ together define a $\tau$-connection on $p^{*} L$.

Let $\mathcal{M}_{X}\left(D_{L}\right) \subset \mathcal{M}_{X}(n)$ denote the subvariety consisting of all $\tau$-connections $(E, D)$ such that the $\tau$-connection $\left(p^{*} L, D_{L}\right)$ is isomorphic to $\left(\bigwedge^{n} E, D\right)$. In other words, $p^{*} L$ is isomorphic to $\bigwedge^{n} E$ and an (hence any) isomorphism takes the $\tau$-connection $D_{L}$ on $p^{*} L$ to the one on $\bigwedge^{n} E$ induced by $D$. Therefore, $\mathcal{M}_{X}\left(D_{L}\right)$ is the analog of a fixed determinant moduli space. The moduli space $\mathcal{M}_{X}\left(D_{L}\right)$ is a reduced irreducible quasi-projective variety over $\mathbb{C}$ of dimension $2\left(n^{2}-1\right)(g-1)+1$ equipped with a natural projection to $\mathbb{C}$.

The isomorphism class of the variety $\mathcal{M}_{X}\left(D_{L}\right)$ does not depend on the choice of $L$ or $D_{L}$.

For another compact connected Riemann surface $Y$ of genus $g$, let $M$ be a line bundle on $Y$ and $D_{M}$ a $\tau$-connection on the pullback of $M$ to $Y \times \mathbb{C}$. Let $\mathcal{M}_{Y}\left(D_{M}\right)$ denote the moduli space of all rank $n$ semistable $\tau$-connections on $Y$ such that the $\tau$-connection induced on the top exterior product coincides with $\left(M, D_{M}\right)$.

The following result is proved in $\S 3$.

Theorem 1.1. If the two varieties $\mathcal{M}_{Y}\left(D_{M}\right)$ and $\mathcal{M}_{X}\left(D_{L}\right)$ are isomorphic, then $X$ is isomorphic to $Y$.

Let $\mathcal{M}_{X}^{D}$ denote the moduli space of all flat connections on $X$ of rank $n$. We prove that there are only finitely many Riemann surfaces $Y$ with the property that the variety $\mathcal{M}_{X}^{D}$ is isomorphic to $\mathcal{M}_{Y}^{D}$, the moduli space of all flat connections of rank $n$ on $Y$. Furthermore, if $X$ is very general, then $\mathcal{M}_{X}^{D}$ is isomorphic to the moduli space of all flat connections of rank $n$ on a Riemann surface $Y$ if and only if $Y$ is isomorphic to $X$ (Theorem 5.2).

Let $\operatorname{Hom}^{r}\left(\pi_{1}(X), \operatorname{GL}(n, \mathbb{C})\right) / \mathrm{GL}(n, \mathbb{C})$ be the quotient of the space of all completely reducible representations of the fundamental group of $X$ in $\operatorname{GL}(n, \mathbb{C})$ by the conjugation action of $\operatorname{GL}(n, \mathbb{C})$ on the representation space. Given a flat vector bundle $E$ over $X$, considering the monodromy of the connection a homomorphism from $\pi_{1}\left(X, x_{0}\right)$ to $\mathrm{GL}\left(E_{x_{0}}\right)$ is obtained. This defines a biholomorphism of the moduli space $\mathcal{M}_{X}^{D}$ with the representation space $\operatorname{Hom}^{r}\left(\pi_{1}(X), \operatorname{GL}(n, \mathbb{C})\right) / \operatorname{GL}(n, \mathbb{C})$. Consequently, the biholomorphism class of the variety $\mathcal{M}_{X}^{D}$ depends only on the topology of $X$. In other words, $\mathcal{M}_{X}^{D}$ is biholomorphic to $\mathcal{M}_{Y}^{D}$ if $X$ and $Y$ are of same genus. Thus the analytic and algebraic isomorphism classes of $\mathcal{M}_{X}^{D}$ are different.

In [Bis02] we investigated the moduli space of rank one connections on a Riemann surface.

\section{Moduli space of Higgs bundle}

As before, let $X$ be a compact connected Riemann surface of genus $g$, with $g \geqslant 3$. A Higgs bundle over $X$ is a pair $(V, \theta)$, where $V$ is a holomorphic vector bundle over $X$ and $\theta$ is a holomorphic section of $K_{X} \otimes \operatorname{End}(V)$. The Higgs bundle $(V, \theta)$ is called stable if for every proper subbundle $F$ of $V$ of positive rank, with $\theta(F) \subset K_{X} \otimes F$, the strict inequality

$$
\operatorname{degree}(F) / \operatorname{rank}(F)<\operatorname{degree}(V) / \operatorname{rank}(V)
$$

is satisfied; if instead the weaker inequality ' $\leqslant$ ' is satisfied then it is called semistable [Hit87].

Fix a line bundle $L$ over $X$ of degree zero and an integer $n \geqslant 2$. Let $\mathcal{M}_{X}^{H}(L)$ denote the moduli space of all semistable Higgs bundles $(V, \theta)$ of rank $n$ over $X$ satisfying the two conditions that $\bigwedge^{n} E \cong L$ and $\operatorname{trace}(\theta)=0[$ Hit87, Sim94a]. 


\section{MOduli SPACE OF $\tau$-CONNECTIONS}

The moduli space $\mathcal{M}_{X}^{H}(L)$ is equipped with a natural action of $\mathbb{C}^{*}$. For any $c \in \mathbb{C}^{*}$, the action of $c$ sends a Higgs bundle $(V, \theta)$ to $(V, c \theta)$. (See [Sim92, Hit87] for properties of this action.)

Let $\omega$ denote the vector field on $\mathcal{M}_{X}^{H}(L)$ defined by the action of $\mathbb{C}^{*}$. In other words, for any point $z \in \mathcal{M}_{X}^{H}(L)$, the tangent vector $\omega(z) \in T_{z} \mathcal{M}_{X}^{H}(L)$ coincides with the image of $1 \in T_{1} \mathbb{C}^{*}$ by the differential of the map $\mathbb{C}^{*} \longrightarrow \mathcal{M}_{X}^{H}(L)$ defined by the orbit of the action passing through $z$.

Let $\mathcal{N}_{X}(L)$ denote the moduli space of (usual) semistable vector bundles $V$ over $X$ of rank $n$ with $\bigwedge^{n} V$ isomorphic to $L$. There is a natural inclusion map

$$
\psi: \mathcal{N}_{X}(L) \longrightarrow \mathcal{M}_{X}^{H}(L)
$$

that sends any $V$ to the Higgs bundle $(V, 0)$. Clearly the above vector field $\omega$ vanishes on $\psi\left(\mathcal{N}_{X}(L)\right)$.

Let $\mathcal{N}_{X}^{s}(L) \subset \mathcal{N}_{X}(L)$ denote the moduli space of all stable vector bundles.

Let $\mathcal{M}_{X}^{H}(L)_{s} \subset \mathcal{M}_{X}^{H}(L)$ denote the smooth locus. So $\mathcal{M}_{X}^{H}(L)_{s}$ is the moduli space of stable Higgs bundles. Note that the action of $\mathbb{C}^{*}$ on $\mathcal{M}_{X}^{H}(L)$ preserves $\mathcal{M}_{X}^{H}(L)_{s}$ (and hence its complement, namely the singular locus). Let $Z(\omega) \subset \mathcal{M}_{X}^{H}(L)_{s}$ denote the subvariety where the vector field $\omega$ vanishes.

Lemma 2.1. The subvariety $Z(\omega)$ of $\mathcal{M}_{X}^{H}(L)_{s}$ has exactly one irreducible component of dimension $\left(n^{2}-1\right)(g-1)$ and all other components are of smaller dimension. This unique component is $\psi\left(\mathcal{N}_{X}^{s}(L)\right)=\psi\left(\mathcal{N}_{X}(L)\right) \cap \mathcal{M}_{X}^{H}(L)_{s}$.

Proof. Take any point $z \in Z(\omega)$. Since $\omega$ vanishes at $z$, we conclude that $z$ must be a fixed point of the $\mathbb{C}^{*}$ action on $\mathcal{M}_{X}^{H}(L)_{s}$. Indeed, by considering translations using the action, the vector field $\omega$ must vanish on the entire orbit of $z$ and hence the orbit is the single point $z$.

Let $\mathcal{N} \subset \mathcal{M}_{X}^{H}(L)_{s}$ be the nilpotent cone that consists of all Higgs bundles $(V, \theta)$ such that $\operatorname{trace}\left(\theta^{j}\right)=0$ for all $j \geqslant 1$. In other words, $\mathcal{N}$ is the inverse image of 0 of the Hitchin map [Hit87, Lau88]. (We will define Hitchin map in $\S 4$ where we actually use it.) The components of $\mathcal{N}$ are parametrized by the conjugacy classes in $\operatorname{sl}(n, \mathbb{C})$ (for the adjoint action of $\operatorname{SL}(n, \mathbb{C})$ ) of the subvariety of nilpotent elements. Each component is of dimension $\left(n^{2}-1\right)(g-1)$. In fact they are Lagrangian subvarieties. The image $\psi\left(\mathcal{N}_{X}^{s}(L)\right)$ is the component of $\mathcal{N}$ that corresponds to $0 \in \operatorname{sl}(n, \mathbb{C})$. Each component of $\mathcal{N}$ is preserved by the $\mathbb{C}^{*}$ action. See [Lau88] for the details.

Since trace $\left((c \theta)^{j}\right)=c^{j} \operatorname{trace}\left(\theta^{j}\right)$, it follows immediately that all the fixed points of the $\mathbb{C}^{*}$ action, namely the points of $Z(\omega)$, are contained in $\mathcal{N}$.

Take any component $\mathcal{N}^{\prime}$ of $\mathcal{N}$ other than $\psi\left(\mathcal{N}_{X}^{s}(L)\right)$. Let $z$ be a point of $\mathcal{N}^{\prime}$ which is not in any other component of $\mathcal{N}$. The Lemma 11.9 of [Sim94b] (see [Sim94b, p. 76]) says that there is a different point $z^{\prime} \in \mathcal{N}^{\prime}, z^{\prime} \neq z$, such that $z$ is in the closure of the orbit (for the $\mathbb{C}^{*}$ action) of $z^{\prime}$. This immediately implies that the action of $\mathbb{C}^{*}$ is non-trivial on the general point of $\mathcal{N}^{\prime}$.

Therefore, the general point of $\mathcal{N}^{\prime}$ is not contained in $Z(\omega)$. On the other hand, we have $\psi\left(\mathcal{N}_{X}^{s}(L)\right) \subset Z(\omega)$ and $Z(\omega) \subset \mathcal{N}$. Since all the components of $\mathcal{N}$ are of dimension $\left(n^{2}-1\right)(g-1)$, this completes the proof of the lemma.

The following lemma implies that any vector field on $\mathcal{M}_{X}^{H}(L)_{s}$ vanishes on the subvariety $\psi\left(\mathcal{N}_{X}^{s}(L)\right)$.

Lemma 2.2. $H^{0}\left(\mathcal{N}_{X}^{s}(L), \psi^{*} T \mathcal{M}_{X}^{H}(L)_{s}\right)=0$.

Proof. The total space of the cotangent bundle $T^{*} \mathcal{N}_{X}^{s}(L)$ sits inside $T \mathcal{M}_{X}^{H}(L)_{s}$ with $\psi$ identified with the zero section. Hence

$$
\psi^{*} T \mathcal{M}_{X}^{H}(L)_{s} \cong T \mathcal{N}_{X}^{s}(L) \oplus T^{*} \mathcal{N}_{X}^{s}(L)
$$




\section{BISWAS}

Consequently, it suffices to show that

$$
H^{0}\left(\mathcal{N}_{X}^{s}(L), T \mathcal{N}_{X}^{s}(L)\right)=0=H^{0}\left(\mathcal{N}_{X}^{s}(L), T^{*} \mathcal{N}_{X}^{s}(L)\right) .
$$

This is well known and follows immediately from the method, initiated in [NR75], of computing cohomology on moduli space using the Hecke transformation. However, we will give the details of the argument.

Let $\mathcal{M}_{X}(n,-1)$ denote the moduli space of all semistable vector bundles over $X$ of rank $n$ and degree -1 . Let $\mathbb{P}$ denote the universal projective bundle over $X \times \mathcal{N}_{X}^{s}(L)$. So $\mathbb{P}$ is the moduli space of triples of the form $(x, E, l)$, where $x \in X, E \in \mathcal{N}_{X}^{s}(L)$, and $l$ is a quotient of $E_{x}$ of dimension one. Let

$$
f: \mathbb{P}--\rightarrow \mathcal{Z} \subset X \times \mathcal{M}_{X}(n,-1)
$$

be the Hecke map (see [NR75] for its definition) where $\mathcal{Z}$ is the fiber bundle over $X$ whose fiber over any $x \in X$ is the moduli space of all semistable vector bundles $V$ over $X$ of rank $n$ and $\bigwedge^{n} V \cong L \otimes \mathcal{O}_{X}(-x)$.

Let $p$ (respectively, $q$ ) denote the obvious projection of $\mathbb{P}$ to $\mathcal{N}_{X}^{s}(L)$ (respectively, $X$ ). We know

$$
T_{(q, p)} \otimes q^{*} K_{X} \cong T_{f}^{*}
$$

on $\mathbb{P}$, where $T_{f}^{*}$ is the relative cotangent bundle for $f$ and $T_{(q, p)}$ is the relative tangent bundle for the projection $(q, p)$ to $X \times \mathcal{N}_{X}^{s}(L)$.

We have $T \mathcal{N}_{X}^{s}(L) \cong \mathrm{R}^{1} p_{*} T_{(q, p)}$ and $T^{*} \mathcal{N}_{X}^{s}(L) \cong p_{*}\left(T_{(q, p)} \otimes q^{*} K_{X}\right)$.

So, $H^{0}\left(\mathcal{N}_{X}^{s}(L), T \mathcal{N}_{X}^{s}(L)\right) \cong H^{1}\left(\mathbb{P}, T_{(q, p)}\right)$ (since $(q, p)$ is a projective fibration). But using $(2.2)$ and taking the direct image to $\mathcal{Z}$,

$$
H^{1}\left(\mathbb{P}, T_{(q, p)}\right) \cong H^{0}(X, T X)=0
$$

as $f$ is also a projective fibration.

Similarly, $H^{0}\left(\mathcal{N}_{X}^{s}(L), T^{*} \mathcal{N}_{X}^{s}(L)\right) \cong H^{0}\left(\mathbb{P}, T_{(q, p)} \otimes q^{*} K_{X}\right)$. Now using $(2.2)$,

$$
H^{0}\left(\mathbb{P}, T_{(q, p)} \otimes q^{*} K_{X}\right) \cong H^{0}\left(\mathcal{Z}, f_{*} T_{f}^{*}\right)=0 .
$$

Indeed, $f_{*} T_{f}^{*}=0$ as $f$ is a projective fibration.

Since $H^{0}\left(\mathcal{N}_{X}^{s}(L), T \mathcal{N}_{X}^{s}(L)\right)=0=H^{0}\left(\mathcal{N}_{X}^{s}(L), T^{*} \mathcal{N}_{X}^{s}(L)\right)$, the proof of the lemma is complete.

Now we are in a position to prove a Torelli type theorem for $\mathcal{M}_{X}^{H}(L)$. Let $Y$ be a compact connected Riemann surface of genus $g$ and $M$ a holomorphic line bundle over $Y$ of degree zero. Let $\mathcal{M}_{Y}^{H}(M)$ denote the moduli space of all semistable Higgs bundles $(V, \theta)$ of rank $n$ over $Y$ with $\bigwedge^{n} V \cong M$ and $\operatorname{trace}(\theta)=0$.

TheOREM 2.3. If the two varieties $\mathcal{M}_{X}^{H}(L)$ and $\mathcal{M}_{Y}^{H}(M)$ are isomorphic, then the Riemann surface $X$ is biholomorphic to $Y$.

Proof. From Lemma 2.2 it follows that for any $\mathbb{C}^{*}$ action on $\mathcal{M}_{X}^{H}(L)$, the subvariety $\psi\left(\mathcal{N}_{X}(L)\right)$ is contained in the fixed point set of the action. On the other hand, Lemma 2.1 implies that there is a $\mathbb{C}^{*}$ action on $\mathcal{M}_{X}^{H}(L)$ such that the only irreducible component of dimension $\left(n^{2}-1\right)(g-1)$ of the fixed point set is $\psi\left(\mathcal{N}_{X}(L)\right)$, and all other components for this action are of strictly smaller dimension. Therefore, the subvariety $\psi\left(\mathcal{N}_{X}(L)\right)$ of $\mathcal{M}_{X}^{H}(L)$ is determined among all the subvarieties by the following two properties:

i) it is irreducible of dimension $\left(n^{2}-1\right)(g-1)$;

ii) for any $\mathbb{C}^{*}$ action on $\mathcal{M}_{X}^{H}(L)$, it is contained in the fixed point set.

In other words, any subvariety of $\mathcal{M}_{X}^{H}(L)$ that satisfies the two conditions must be $\psi\left(\mathcal{N}_{X}(L)\right)$. 


\section{MOduli SPACE OF $\tau$-CONNECTIONS}

A theorem due to Kouvidakis and Pantev [KP95, p. 229, Theorem E] says that if the moduli space $\mathcal{N}_{X}(L)$ is isomorphic to the moduli space $\mathcal{M}_{Y}(M)$ of all fixed determinant semistable vector bundles on $Y$, then $X$ is isomorphic to $Y$. This completes the proof of the theorem.

In the next section we will use Theorem 2.3 to prove Theorem 1.1 stated in $\S 1$.

\section{Proof of Theorem 1.1}

Fix a holomorphic connection $\nabla^{L}$ on $L$. Note that a holomorphic connection on a Riemann surface is automatically flat. Consider all holomorphic connections of the form $(V, \nabla)$ on $X$, where $V$ is a holomorphic vector bundle of rank $n$ with $\bigwedge^{n} V \cong L$ and $\nabla$ is a holomorphic connection on $V$ such that the above isomorphism takes the connection $\nabla^{L}$ to the one on $\bigwedge^{n} V$ induced by $\nabla$. Let $\mathcal{M}_{X}^{D}(L)$ denote the moduli space of all connections of this type [Sim94a]. Note that for a different choice of $\nabla^{L}$, the corresponding moduli space is canonically isomorphic to $\mathcal{M}_{X}^{D}(L)$. Therefore, the moduli space is independent of the choice of $\nabla^{L}$.

Consider the moduli space of $\tau$-connections $\mathcal{M}_{X}\left(D_{L}\right)$ defined in $\S 1$. We recall from [Sim94a] that there is a natural projection

$$
\gamma: \mathcal{M}_{X}\left(D_{L}\right) \longrightarrow \mathbb{C}
$$

such that the fiber over any $c \in \mathbb{C}^{*}$ is isomorphic to the moduli space $\mathcal{M}_{X}^{D}(L)$ and the fiber over zero is the moduli space of Higgs bundles $\mathcal{M}_{X}^{H}(L)$.

Lemma 3.1. $H^{0}\left(\mathcal{M}_{X}^{D}(L), \mathcal{O}_{\mathcal{M}_{X}^{D}(L)}\right)=\mathbb{C}$.

This lemma will be proved in the next section. In this section we will assume Lemma 3.1 and prove Theorem 1.1.

Now, Lemma 3.1 implies that for any $c \in \mathbb{C}^{*}$, there is no non-constant function on the fiber $\gamma^{-1}(c)$, where $\gamma$ is the map in (3.1). Consequently, any function on $\mathcal{M}_{X}\left(D_{L}\right)$ is the pullback, using $\gamma$, of a function on $\mathbb{C}$. In other words, considering the natural map

$$
\mathcal{M}_{X}\left(D_{L}\right) \longrightarrow \operatorname{Spec}\left(\Gamma\left(\mathcal{M}_{X}\left(D_{L}\right)\right)\right)
$$

where $\Gamma\left(\mathcal{M}_{X}\left(D_{L}\right)\right)$ is the algebra of global functions on $\mathcal{M}_{X}\left(D_{L}\right)$, the fibration defined by $\gamma$ is recovered.

If all the fibers of $\gamma$ are isomorphic, then taking any fiber of $(3.2)$ we get $\mathcal{M}_{X}^{H}(L)$. If $\mathcal{M}_{X}^{H}(L)$ is not isomorphic to $\mathcal{M}_{X}^{D}(L)$, then we obtain $\mathcal{M}_{X}^{H}(L)$ as the unique fiber of the map in (3.2) which is different from all other fibers (which are themselves isomorphic).

In view of Lemma 2.3, this observation completes the proof of Theorem 1.1.

\section{Proof of Lemma 3.1}

Let $U \subset \mathcal{M}_{X}^{D}(L)$ be the (non-empty) Zariski open subset consisting of all pairs $(V, \nabla)$ such that the underlying holomorphic vector bundle $V$ is stable. To prove Lemma 3.1 it suffices to show

$$
H^{0}\left(U, \mathcal{O}_{U}\right)=\mathbb{C},
$$

that is, $U$ does not admit any non-constant function.

As before, let $\mathcal{N}_{X}^{s}(L)$ denote the moduli space of stable vector bundles of rank $n$ and determinant $L$. So we have a natural projection

$$
\beta: U \longrightarrow \mathcal{N}_{X}^{s}(L)
$$




\section{BISWAS}

that sends any $(V, \nabla)$ to the underlying vector bundle $V$. The fiber of $\beta$ over a vector bundle $V \in \mathcal{N}_{X}^{s}(L)$ is an affine space for the vector space

$$
H^{0}\left(X, K_{X} \otimes \operatorname{ad}(V)\right) \cong T_{V}^{*} \mathcal{N}_{X}^{s}(L) .
$$

Indeed, any two connections on $V$ with a fixed connection on $\bigwedge^{n} V$ differ by a section of $K_{X} \otimes \operatorname{ad}(V)$. In other words, $U$ is a $T^{*} \mathcal{N}_{X}^{s}(L)$-torsor.

Let $\Theta$ denote the generalized theta line bundle over $\mathcal{N}_{X}^{s}(L)$. In other words, $\Theta$ is the positive generator of $\operatorname{Pic}\left(\mathcal{N}_{X}^{s}(L)\right) \cong \mathbb{Z}$.

Consider the Atiyah exact sequence (see [AtiS7])

$$
0 \longrightarrow \mathcal{O} \longrightarrow \operatorname{At}(\Theta):=\operatorname{Diff}^{1}(\Theta, \Theta) \longrightarrow T \mathcal{N}_{X}^{s}(L) \longrightarrow 0,
$$

where the surjective map is the symbol map of differential operator. Let

$$
0 \longrightarrow T^{*} \mathcal{N}_{X}^{s}(L) \stackrel{\sigma}{\longrightarrow} \operatorname{At}(\Theta)^{*} \stackrel{\alpha}{\longrightarrow} \mathcal{O} \longrightarrow 0
$$

be the dual sequence.

The subvariety $\alpha^{-1}(1)$ of the total space of $\operatorname{At}(\Theta)^{*}$ defined by the inverse image of the image of the constant section 1 of $\mathcal{O}$ will be denoted by $\operatorname{Conn}(\Theta)$.

Let

$$
\phi: \operatorname{Conn}(\Theta) \longrightarrow \mathcal{N}_{X}^{s}(L)
$$

be the natural projection. Clearly, the map $\phi$ makes $\operatorname{Conn}(\Theta)$ a $T^{*} \mathcal{N}_{X}^{s}(L)$-torsor. The space of all holomorphic sections of $\phi$ over an open subset $W$ is in bijective correspondence with the space of all holomorphic connections on $\left.\Theta\right|_{W}$ [AtiS7].

It is known that the variety $\operatorname{Conn}(\Theta)$ is isomorphic to $U$. The collection of all isomorphism classes of $T^{*} \mathcal{N}_{X}^{s}(L)$-torsors are parametrized by $H^{1}\left(\mathcal{N}_{X}^{s}(L), T^{*} \mathcal{N}_{X}^{s}(L)\right)$. Let $A_{1}$ and $A_{2}$ be two such torsors corresponding to cohomology classes $\omega_{1}$ and $\omega_{2}$ respectively. Then the total space of $A_{1}$ is isomorphic to the total space of $A_{2}$ if $\omega_{1}=c \omega_{2}$ for some $c \in \mathbb{C}^{*}$. Since

$$
\operatorname{dim} H^{1}\left(\mathcal{N}_{X}^{s}(L), T^{*} \mathcal{N}_{X}^{s}(L)\right)=1,
$$

the total spaces of any two non-trivial $T^{*} \mathcal{N}_{X}^{s}(L)$-torsors are isomorphic. It is easy to see that both the torsors $U$ and $\operatorname{Conn}(\Theta)$ are non-trivial. In [ZT86] and [BR98] it is proved that $U$ and $\operatorname{Conn}(\Theta)$ are isomorphic as $T^{*} \mathcal{N}_{X}^{s}(L)$-torsors.

Therefore, the following lemma proves (4.1).

Lemma 4.1. $H^{0}\left(\operatorname{Conn}(\Theta), \mathcal{O}_{\operatorname{Conn}(\Theta)}\right)=\mathbb{C}$.

Proof. To prove the lemma, let $\mathbb{P A t}(\Theta)$ be the projective bundle over $\mathcal{N}_{X}^{s}(L)$ consisting of all lines in $\operatorname{At}(\Theta)^{*}$. Let $\mathbb{P} T$ denote the projectivization of the tangent bundle $T \mathcal{N}_{X}^{s}(L)$, that is, the space of all lines in $T^{*} \mathcal{N}_{X}^{s}(L)$. Using the homomorphism $\sigma$ in (4.3), we have $\mathbb{P} T$ as a subvariety of $\mathbb{P A t}(\Theta)$. The complement coincides with $\operatorname{Conn}(\Theta)$. Therefore,

$$
H^{0}\left(\operatorname{Conn}(\Theta), \mathcal{O}_{\operatorname{Conn}(\Theta)}\right) \cong H^{0}\left(\mathbb{P A t}(\Theta), \mathcal{O}_{\mathbb{P A t}(\Theta)}(* \mathbb{P} T)\right),
$$

where $H^{0}\left(\mathbb{P A t}(\Theta), \mathcal{O}_{\mathbb{P A t}(\Theta)}(* \mathbb{P} T)\right)$ is the direct limit of $H^{0}\left(\mathbb{P A t}(\Theta), \mathcal{O}_{\mathbb{P A t}(\Theta)}(k \mathbb{P} T)\right)$ with $k \in \mathbb{N}$.

Note that the line bundle $\mathcal{O}_{\mathbb{P A t}(\Theta)}(\mathbb{P} T)$ is canonically identified with the tautological line bundle $\mathcal{O}_{\mathbb{P A t}(\Theta)}(1)$. So, for any $k \geqslant 0$,

$$
H^{0}\left(\mathbb{P A t}(\Theta), \mathcal{O}_{\mathbb{P A t}(\Theta)}(k \mathbb{P} T)\right) \cong H^{0}\left(\mathcal{N}_{X}^{s}(L), \operatorname{Sym}^{k} \operatorname{At}(\Theta)\right),
$$

where $\mathrm{Sym}^{k}$ denotes the $k$ th symmetric power.

From (4.2) we have an exact sequence

$$
0 \longrightarrow \operatorname{Sym}^{k-1} \operatorname{At}(\Theta) \longrightarrow \operatorname{Sym}^{k} \operatorname{At}(\Theta) \longrightarrow \operatorname{Sym}^{k} T_{\mathcal{N}}^{s}(L) \longrightarrow 0
$$


for each $k \geqslant 1$. In view of (4.4) and (4.5), to prove the lemma it suffices to show that the coboundary homomorphism (for the exact sequence (4.6))

$$
b_{k}: H^{0}\left(\mathcal{N}_{X}^{s}(L), \operatorname{Sym}^{k} T \mathcal{N}_{X}^{s}(L)\right) \longrightarrow H^{1}\left(\mathcal{N}_{X}^{s}(L), \operatorname{Sym}^{k-1} \operatorname{At}(\Theta)\right)
$$

is injective for each $k \geqslant 1$. Let

$$
\operatorname{Sym}^{k-1}\left(\sigma^{*}\right): \operatorname{Sym}^{k-1} \operatorname{At}(\Theta) \longrightarrow \operatorname{Sym}^{k-1} T \mathcal{N}_{X}^{s}(L)
$$

be the symmetric power of the projection $\sigma^{*}: \operatorname{At}(\Theta) \longrightarrow T \mathcal{N}_{X}^{s}(L)$ in (4.2). Let

$$
f_{k}:=\operatorname{Sym}^{k-1}\left(\sigma^{*}\right) \circ b_{k}: H^{0}\left(\mathcal{N}_{X}^{s}(L), \operatorname{Sym}^{k} T \mathcal{N}_{X}^{s}(L)\right) \longrightarrow H^{1}\left(\mathcal{N}_{X}^{s}(L), \operatorname{Sym}^{k-1} T \mathcal{N}_{X}^{s}(L)\right)
$$

be the composition homomorphism constructed in an obvious way. To prove that the above coboundary homomorphism $b_{k}, k \geqslant 1$, is injective, it is enough to show that $f_{k}$ in (4.7) is injective for each $k \geqslant 1$.

Since the natural projection

$$
\phi^{1}: T^{*} \mathcal{N}_{X}^{s}(L) \longrightarrow \mathcal{N}_{X}^{s}(L)
$$

is an affine fibration, all the higher direct images vanish. Consequently, we have

$$
H^{i}\left(T^{*} \mathcal{N}_{X}^{s}(L), \mathcal{O}\right) \cong H^{i}\left(\mathcal{N}_{X}^{s}(L), \phi_{*}^{1} \mathcal{O}\right) \cong \bigoplus_{j \geqslant 0}^{\infty} H^{i}\left(\mathcal{N}_{X}^{s}(L), \operatorname{Sym}^{j} T \mathcal{N}_{X}^{s}(L)\right)
$$

for each $i \geqslant 0$. Now, to describe $H^{i}\left(T^{*} \mathcal{N}_{X}^{s}(L), \mathcal{O}\right)$ we will use the Hitchin map.

Let

$$
\mathcal{H}: T^{*} \mathcal{N}_{X}^{s}(L) \longrightarrow \mathcal{V}:=\bigoplus_{i=1}^{n} H^{0}\left(X, K_{X}^{\otimes i}\right)
$$

be the Hitchin map that sends any $(E, \theta)$ to $\sum_{i=1}^{n} \operatorname{trace}\left(\theta^{i}\right)$. The general fiber of $\mathcal{H}$ is a complement, in some abelian variety (called a Prym variety), of a subvariety of codimension at least three (see [BNR89, Hit87]). Hence any function on $T^{*} \mathcal{N}_{X}^{s}(L)$ descends to a function on the vector space $\mathcal{V}$. The space of all functions on $\mathcal{V}$ that vanish at 0 is identified with

$$
\mathcal{C}:=d H^{0}\left(\mathcal{V}, \mathcal{O}_{\mathcal{V}}\right) \subset H^{0}\left(\mathcal{V}, T^{*} \mathcal{V}\right)
$$

the space of all exact algebraic one-forms on $\mathcal{V}$, by sending any function $f$ to $d f$.

Combining these, and setting $i=0$ in (4.8), we have an isomorphism

$$
\delta: \bigoplus_{k=1}^{\infty} H^{0}\left(\mathcal{N}_{X}^{s}(L), \operatorname{Sym}^{k} T \mathcal{N}_{X}^{s}(L)\right) \cong H^{0}\left(T^{*} \mathcal{N}_{X}^{s}(L), \mathcal{O}\right) \longrightarrow \mathcal{C}
$$

that sends any section $s \in H^{0}\left(\mathcal{N}_{X}^{s}(L), \operatorname{Sym}^{k} T \mathcal{N}_{X}^{s}(L)\right)$ to $d f$, where $f$ is the function on $\mathcal{V}$ defined by $s$.

Let $T_{\mathcal{H}}$ denote the relative tangent bundle on $T^{*} \mathcal{N}_{X}^{s}(L)$ for the Hitchin map. Using the symplectic structure on $T^{*} \mathcal{N}_{X}^{s}(L)$, the pulled back bundle $\mathcal{H}^{*} T^{*} \mathcal{V}$ is identified with $T_{\mathcal{H}}$ over the general fiber. This follows from the fact that $\mathcal{H}$ defines a completely integrable system. Consequently, $H^{0}\left(\mathcal{V}, T^{*} \mathcal{V}\right)$ is identified with a subspace of the space of sections of $T_{\mathcal{H}}$. So we have constructed an injective homomorphism

$$
\iota: \mathcal{C}=\bigoplus_{k=1}^{\infty} \delta\left(H^{0}\left(\mathcal{N}_{X}^{s}(L), \operatorname{Sym}^{k} T \mathcal{N}_{X}^{s}(L)\right)\right) \longrightarrow H^{0}\left(T^{*} \mathcal{N}_{X}^{s}(L), T_{\mathcal{H}}\right)
$$

from the space of all exact algebraic forms.

Any $\omega \in H^{1}\left(\mathcal{N}_{X}^{s}(L), T^{*} \mathcal{N}_{X}^{s}(L)\right)$ gives a homomorphism

$$
H^{0}\left(\mathcal{N}_{X}^{s}(L), \operatorname{Sym}^{k} T \mathcal{N}_{X}^{s}(L)\right) \longrightarrow H^{1}\left(\mathcal{N}_{X}^{s}(L), \operatorname{Sym}^{k} T \mathcal{N}_{X}^{s}(L) \otimes T^{*} \mathcal{N}_{X}^{s}(L)\right)
$$




\section{BISWAS}

by cupping with $\omega$. Now contracting $T^{*} \mathcal{N}_{X}^{s}(L)$ with its dual $T^{*} \mathcal{N}_{X}^{s}(L)$ a homomorphism

$$
H^{0}\left(\mathcal{N}_{X}^{s}(L), \operatorname{Sym}^{k} T \mathcal{N}_{X}^{s}(L)\right) \longrightarrow H^{1}\left(\mathcal{N}_{X}^{s}(L), \operatorname{Sym}^{k-1} T \mathcal{N}_{X}^{s}(L)\right)
$$

is obtained. The homomorphism $f_{k}$ in (4.7) coincides with the homomorphism in (4.11) for $\omega=$ $k c_{1}(\Theta)$.

Consider the homomorphism

$$
\Phi: H^{0}\left(T^{*} \mathcal{N}_{X}^{s}(L), T_{\mathcal{H}}\right) \longrightarrow H^{1}\left(T^{*} \mathcal{N}_{X}^{s}(L), \mathcal{O}\right)
$$

constructed using the combination of the cup product defined by

$$
c_{1}\left(\left(\phi^{1}\right)^{*} \Theta\right) \in H^{1}\left(T^{*} \mathcal{N}_{X}^{s}(L), T^{*} T^{*} \mathcal{N}_{X}^{s}(L)\right)
$$

and the natural duality pairing $T_{\mathcal{H}} \otimes T^{*} T^{*} \mathcal{N}_{X}^{s}(L) \longrightarrow \mathcal{O}_{T^{*} \mathcal{N}_{X}^{s}(L)}$. Since $c_{1}\left(\left(\phi^{1}\right)^{*} \Theta\right)=\left(\phi^{1}\right)^{*} c_{1}(\Theta)$, from the above description of $f_{k}$ using (4.11) it follows immediately that $k \Phi \circ \iota \circ \delta\left(w_{k}\right)$, for any $w_{k} \in H^{0}\left(\mathcal{N}_{X}^{s}(L), \operatorname{Sym}^{k} T \mathcal{N}_{X}^{s}(L)\right)$, coincides with the image of $f_{k}\left(w_{k}\right)$ in $H^{1}\left(T^{*} \mathcal{N}_{X}^{s}(L), \mathcal{O}\right)$ by $(4.8)$. Therefore, to prove that each $f_{i}, i \geqslant 1$, is injective, it suffices to show that the restriction $\left.\Phi\right|_{\iota(\mathcal{C})}$ is injective.

The pullback of $\Theta$ to any fiber of the Hitchin map remains ample [BNR89]. For an ample line bundle $L$ on an abelian variety $A$, the homomorphism $H^{0}(A, T A) \longrightarrow H^{1}\left(A, \mathcal{O}_{A}\right)$ defined by cupping with $c_{1}(L) \in H^{1}\left(A, T^{*} A\right.$ ) (as in (4.11)) is an isomorphism. In particular, this homomorphism for the restriction of $\Theta$ to a Prym variety (general fiber for the Hitchin map on the moduli space of Higgs bundles) is injective. Using this together with the earlier remark that the general fiber of $\mathcal{H}$ is the complement in a Prym variety (which is an abelian variety) of some subvariety of codimension at least three, it follows immediately that the homomorphism $\left.\Phi\right|_{\iota(\mathcal{C})}$ is injective. Indeed, given any non-zero exact one-form $\nu \in \mathcal{C} \backslash\{0\}$, take $v \in \mathcal{V}$ such that $\nu(v) \neq 0$ and the fiber $\mathcal{H}^{-1}(v)$ is the complement (in the Prym variety) of a subvariety of codimension at least three. From the earlier remark that the homomorphism $H^{0}(A, T A) \longrightarrow H^{1}\left(A, \mathcal{O}_{A}\right)$ is injective it follows that the image of $\nu$ by the composition of the homomorphism $\Phi \circ \iota: \mathcal{C} \longrightarrow H^{1}\left(T^{*} \mathcal{N}_{X}^{s}(L), \mathcal{O}\right)$ with the restriction homomorphism

$$
H^{1}\left(T^{*} \mathcal{N}_{X}^{s}(L), \mathcal{O}\right) \longrightarrow H^{1}\left(\mathcal{H}^{-1}(v), \mathcal{O}\right)
$$

is non-zero. Consequently, the homomorphism $\left.\Phi\right|_{\iota(\mathcal{C})}$ in (4.12) must be injective. This completes the proof of the lemma.

It was noted earlier that Lemma 4.1 proves Lemma 3.1. Therefore, the proof of Lemma 3.1 is complete.

In the next section we will prove a Torelli type theorem for the moduli space of flat connections on $X$.

\section{The moduli space of flat connections}

As before, let $X$ be a compact connected Riemann surface of genus $g$, with $g \geqslant 3$. Let $\mathcal{M}_{X}^{D}$ denote the moduli space of all pairs of the form $(V, \nabla)$, where $V$ is a holomorphic vector bundle of rank $n$ over $X$ and $\nabla$ is a holomorphic connection on $V$. As we noted earlier, $\nabla$ is automatically flat. The moduli space $\mathcal{M}_{X}^{D}$ is irreducible.

Let $\operatorname{Pic}^{0}(X)$ denote the Jacobian of $X$ parametrizing isomorphism classes of all degree zero line bundles. We have a surjective algebraic morphism

$$
F: \mathcal{M}_{X}^{D} \longrightarrow \operatorname{Pic}^{0}(X)
$$

that sends any flat connection $(V, \nabla)$ to the line bundle $\bigwedge^{n} V$. 


\section{MOduli SPACE OF $\tau$-CONNECTIONS}

Lemma 5.1. Let $A$ be a complex abelian variety and

$$
f: \mathcal{M}_{X}^{D} \longrightarrow A
$$

an algebraic morphism. Then there is a unique algebraic morphism

$$
f_{0}: \operatorname{Pic}^{0}(X) \longrightarrow A
$$

satisfying the condition $f_{0} \circ F=f$, where $F$ is defined in (5.1).

Proof. Let $E$ be a stable vector bundle of rank $n$ and degree zero over $X$. Note that any holomorphic connection on $E$ is irreducible. Indeed, if $F$ is a subbundle of $E$ preserved by a holomorphic connection $\nabla$ on $E$, then degree $(F)=0$, as $F$ admits a holomorphic connection. This contradicts stability of $E$ if $F$ is of positive rank.

Let $\mathcal{D}(E)$ denote the space of all holomorphic connections on $E$. This space $\mathcal{D}(E)$ is an affine space for the vector space $H^{0}\left(X, K_{X} \otimes \operatorname{End}(E)\right)$. In particular, $\mathcal{D}(E)$ is a rational variety of dimension $n^{2}(g-1)+1$. So the restriction of $f$ (in the statement of the lemma) to $\mathcal{D}(E)$ is a constant map, as there are no non-constant maps from a rational variety to an abelian variety.

Let $\mathcal{M}_{X}(n)$ denote the moduli space of all stable vector bundles over $X$ of rank $n$ and degree zero. For a non-empty Zariski open subset of the moduli space $\mathcal{M}_{X}^{D}$ the underlying vector bundle over $X$ is stable. In other words, there is a non-empty Zariski open subset $U \subset \mathcal{M}_{X}(n)$ and a map

$$
\psi: U \longrightarrow \mathcal{M}_{X}(n)
$$

defined by $(V, \nabla) \longmapsto V$. For any $E \in \mathcal{M}_{X}(n)$, the fiber $\psi^{-1}(E)$ is $\mathcal{D}(E)$.

The above observation that the restriction of $f$ to $\mathcal{D}(E)$ is a constant map immediately implies that there is a map

$$
\psi_{0}: \mathcal{M}_{X}(n) \longrightarrow A
$$

such that

$$
\psi_{0} \circ \psi=f
$$

over $U$. Let

$$
F_{0}: \mathcal{M}_{X}(n) \longrightarrow \operatorname{Pic}^{0}(X)
$$

be the map that sends any stable vector bundle $V$ to $\bigwedge^{n} V$. So, $F_{0} \circ \psi=F$ over $U$, where $\psi$ and $F$ are defined in (5.2) and (5.1) respectively.

For any degree zero line bundle $L$ over $X$, let $\mathcal{N}_{X}^{s}(L)$ denote the moduli space of all stable vector bundles $V$ of rank $n$ over $X$ with $\bigwedge^{n} V \cong L$. So, $\mathcal{N}_{X}^{s}(L)$ is identified with $F_{0}^{-1}(L)$.

Fix a point $x_{0} \in X$. Let $W$ be a stable vector bundle of rank $n$ over $X$ such that $\bigwedge^{n} W \cong$ $L \otimes \mathcal{O}_{X}\left(x_{0}\right)$. Let $\mathbb{P}\left(W_{x_{0}}\right)$ be the projective space parametrizing all one-dimensional quotients of the fiber $W_{x_{0}}$. For any quotient $\xi \in \mathbb{P}\left(W_{x_{0}}\right)$, consider the vector bundle $W_{\xi}$ over $X$ of rank $n$ defined by the exact sequence

$$
0 \longrightarrow W_{\xi} \longrightarrow W \longrightarrow \xi \longrightarrow 0 .
$$

Clearly, $\bigwedge^{n} W_{\xi} \cong L$. It is easy to check that the vector bundle $W_{\xi}$ is stable (see [BBGN02, p. 563, Lemma 2]).

Consequently, the projective space $\mathbb{P}\left(W_{x_{0}}\right)$ is realized as a subvariety of $\mathcal{N}_{X}^{s}(L)$ by sending any $\xi \in \mathbb{P}\left(W_{x_{0}}\right)$ to $W_{\xi}$ (see [BBGN02, p. 564, Lemma 3]). The restriction of the map $\psi_{0}$ (defined in (5.3)) to $\mathbb{P}\left(W_{x_{0}}\right) \subset \mathcal{N}_{X}^{s}(L)$ must be a constant map, as $A$ being an abelian variety there are no non-constant maps to it from a projective space.

From the above observation that $\left.\psi_{0}\right|_{\mathbb{P}\left(W_{x_{0}}\right)}$ is a constant map it follows that $\left.\psi_{0}\right|_{\mathcal{N}_{X}^{s}(L)}$ itself is a constant map. To prove this first recall that $\operatorname{Pic}\left(\mathcal{N}_{X}^{s}(L)\right) \cong \mathbb{Z}[\mathrm{DN} 89$, p. 55, Théoréme $\mathrm{B}]$. 


\section{BISWAS}

This implies that if the restriction of $\psi_{0}$ to $\mathcal{N}_{X}^{s}(L)$ is not a constant map then the pullback $\psi_{0}^{*} \zeta$ must be an ample line bundle, where $\zeta$ is a very ample line bundle over $A$. Indeed, in that case

$$
\operatorname{dim} H^{0}\left(\mathcal{N}_{X}^{s}(L), \psi_{0}^{*} \zeta\right)>1
$$

and hence $\psi_{0}^{*} \zeta$ must be ample as $\operatorname{Pic}\left(\mathcal{N}_{X}^{s}(L)\right) \cong \mathbb{Z}$ and $\mathcal{N}_{X}^{s}(L)$ does not admit any non-constant holomorphic function. On the other hand, the restriction of $\psi_{0}^{*} \zeta$ to $\mathbb{P}\left(W_{x_{0}}\right)$ is the trivial line bundle. Hence the restriction of $\psi_{0}$ to $\mathcal{N}_{X}^{s}(L)$ must be a constant map.

Consequently, there is an algebraic map

$$
f_{0}: \operatorname{Pic}^{0}(X) \longrightarrow A
$$

such that $\psi_{0}=f_{0} \circ F_{0}$ over $\mathcal{M}_{X}(n)$, where $F_{0}$ and $\psi_{0}$ are defined in (5.5) and (5.3) respectively. Note that as the map $F_{0}$ is surjective, the identity $\psi_{0}=f_{0} \circ F_{0}$ uniquely determines $f_{0}$.

So we have $f_{0} \circ F_{0} \circ \psi=f$ over $U$, as $\psi_{0} \circ \psi=f$ (see (5.4)).

Finally, since $F_{0} \circ \psi=F$ and $U$ is Zariski dense in $\mathcal{M}_{X}^{D}$ the proof of the lemma is complete.

The Torelli theorem for Riemann surfaces says that a connected Riemann surface is determined by its Jacobian equipped with the principal polarization defined by a theta divisor. Lemma 5.1 says that $\mathcal{M}_{X}^{D}$ determines the Jacobian $\operatorname{Pic}^{0}(X)$. But this does not quite determine $X$, as two different Riemann surfaces may have isomorphic Jacobians (in that case the principal polarizations have to be different by the Torelli theorem for Riemann surfaces). However, a result due to Narasimhan and Nori says that there can only be finitely many Riemann surfaces sharing a common Jacobian. More precisely, there are, up to isomorphism, only finitely many compact connected Riemann surfaces having a given abelian variety as their Jacobian [NN81, p. 125, Corollary 1.2].

If $X$ is a very general Riemann surface, then the rank of the Néron-Severi group $\mathrm{NS}\left(\operatorname{Pic}^{0}(X)\right)$ is one [BN97, p. 711, Theorem 5.1]. This means that there is a countable union of proper closed algebraic subvarieties $B$ of the moduli space of complex curves of genus $g$ such that for any Riemann

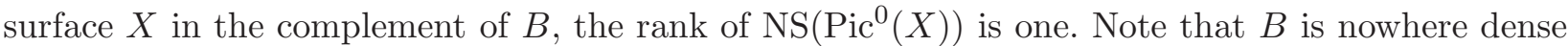
for the Euclidean topology on the moduli space of curves. If the rank of $\operatorname{NS}\left(\operatorname{Pic}^{0}(X)\right)$ is one, then $\operatorname{Pic}^{0}(X)$ clearly can have at most one principal polarization. By a very general Riemann surface we will mean one in the complement of $B$ in the moduli space.

Lemma 5.1 and the above results combine together to give the following theorem.

TheOREM 5.2. There are, up to isomorphism, only finitely many connected Riemann surfaces $Y$ with the property that the moduli space of rank $n$ holomorphic connections on $Y$ is isomorphic to $\mathcal{M}_{X}^{D}$.

If $X$ is a very general Riemann surface, then the condition that $\mathcal{M}_{X}^{D}$ is isomorphic to the moduli space of all rank $n$ holomorphic connections on $Y$ implies that the Riemann surface $Y$ is isomorphic to $X$.

As noted in $\S 1$, the biholomorphism class of $\mathcal{M}_{X}^{D}$ depends only on the genus of $X$. In other words, it is independent of the complex structure of $X$.

Remark 5.3. We will give a brief outline of an argument that $\mathcal{M}_{X}^{D}(L)$ (defined earlier as the moduli space of all connections with a fixed connection on the fixed top exterior power) determines the Jacobian of $X$. Consider the smooth locus of $U$ of $\mathcal{M}_{X}^{D}(L)$. Let $U^{\prime} \subset U$ be the Zariski open subset consisting of all flat connections such that the underlying vector bundle is stable. The natural projection of $U^{\prime}$ to $\mathcal{N}_{X}^{s}(L)$ makes $U^{\prime}$ a Zariski locally trivial fiber bundle over $\mathcal{N}_{X}^{s}(L)$ with $\mathbb{C}^{(g-1)\left(n^{2}-1\right)}$ as the fiber, and the codimension of the complement $U \backslash U^{\prime}$ is at least three. Therefore, the intermediate Jacobian $J^{2}\left(U^{\prime}\right)$ is isomorphic to $J^{2}\left(\mathcal{N}_{X}^{s}(L)\right.$ ) (respectively, $J^{2}(U)$ ) with the isomorphism obtained from the projection (respectively, inclusion) map. (See [BZ98, p. 101] and [EV88, p. 57] 


\section{MOduli SPACE OF $\tau$-CONNECTIONS}

for the intermediate Jacobian of a smooth quasi-projective variety.) On the other hand, $J^{2}\left(\mathcal{N}_{X}^{s}(L)\right)$ is isomorphic to $\operatorname{Pic}^{0}(X)$. Note that

$$
\operatorname{dim} H^{i}\left(\mathcal{N}_{X}^{s}(L), \mathbb{Q}\right)= \begin{cases}0 & \text { if } i=1, \\ 1 & \text { if } i=2, \\ 2 g & \text { if } i=3,\end{cases}
$$

(see $[\operatorname{Kir} 96])$, which implies that $\operatorname{dim} J^{2}\left(\mathcal{N}_{X}^{s}(L)\right)=g$. A natural map from $\operatorname{Pic}^{0}(X)$ to $J^{2}\left(\mathcal{N}_{X}^{s}(L)\right)$ is constructed as follows. Fix a point $x_{0} \in X$ and let $\mathcal{U}$ be the pullback to $\mathcal{N}_{X}^{s}(L) \times \operatorname{Pic}^{0}(X)$ of the universal projective bundle over $X \times \mathcal{M}_{X}(n)$ by the map defined by $(E, \xi) \longmapsto\left(x_{0}, E \otimes \xi\right)$. Considering $\mathcal{U}$ as a family of projective bundles over $\mathcal{N}_{X}^{s}(L)$ parametrized by $\operatorname{Pic}^{0}(X)$ the second characteristic class of a projective bundle defines a map

$$
\varphi: \operatorname{Pic}^{0}(X) \longrightarrow \mathbb{H}_{\mathcal{D}}^{4}\left(\mathcal{N}_{X}^{s}(L)\right)
$$

to the Deligne-Beilinson cohomology; the map $\varphi$ is known as the Abel-Jacobi map (see [EV88, p. 85, Corollary 7.7] and [EV88, p. 86, Theorem 7.11]). The map $\varphi$ induces a map

$$
\varphi^{\prime}: \operatorname{Pic}^{0}(X) \longrightarrow J^{2}\left(\mathcal{N}_{X}^{s}(L)\right)
$$

defined by $\theta \longmapsto \varphi(\theta)-\varphi(0)$, where 0 is the identity element of $\operatorname{Pic}^{0}(X)$. For a different choice of the base point $x_{0}$, the map $\varphi^{\prime}$ simply gets translated. Using this isomorphism $\mathcal{M}_{X}^{D}(L)$ determines $\operatorname{Pic}^{0}(X)$.

\section{ACKNOWLEDGEMENTS}

The proof of Theorem 2.3 presented here is due to S. Ramanan. The author is very grateful to him for communicating the proof. The author is very grateful to David Ben-Zvi for useful discussions on $\tau$-connections. The author thanks the referee for pointing out an inaccuracy in the earlier version.

\section{REFERENCES}

AtiS7 M. F. Atiyah, Complex analytic connections in fibre bundles, Trans. Amer. Math. Soc. 85 (1957), 181-207.

BNR89 A. Beauville, M. S. Narasimhan and S. Ramanan, Spectral curves and the generalised theta divisor, J. Reine Angew. Math. 398 (1989), 169-179.

Bis02 I. Biswas, Differential operators on a polarized abelian variety, Trans. Amer. Math. Soc. 354 (2002), 3883-3891.

BN97 I. Biswas and M. S. Narasimhan, Hodge classes of moduli spaces of parabolic vector bundles over the general curve, J. Algebraic Geom. 6 (1997), 697-715.

BR98 I. Biswas and N. Raghavendra, Curvature of the determinant bundle and the Kähler form over the moduli of parabolic bundles for a family of pointed curves, Asian J. Math. 2 (1998), 303-324.

BBGN02 I. Biswas, L. Brambila-Paz, T. L. Gómez and P. E. Newstead, Stability of the Picard bundle, Bull. London Math. Soc. 34 (2002), 561-568.

BZ98 J.-L. Brylinski and S. Zucker, An overview of recent advances in Hodge theory, in Complex manifolds (Springer-Verlag, Berlin, 1998), 39-142.

DN89 J.-M. Drezet and M. S. Narasimhan, Groupe de Picard des variétés de modules de fibrés semistables sur les courbes algébriques, Invent. Math. 97 (1989), 53-94.

EV88 H. Esnault and E. Viehweg, Deligne-Beilinson cohomology, in Beilinson's conjectures on special values of L-functions, Perspect. Math., vol. 4 (Academic Press, Boston, MA, 1988), 43-91.

Hit87 N. J. Hitchin, Stable bundles and integrable systems, Duke Math. J. 54 (1987), 91-114. 


\section{MOdULi SPACE OF $\tau$-CONNECTIONS}

Kir96 F. Kirwan, On the homology of compactifications of moduli spaces of vector bundles over a Riemann surface, Proc. London Math. Soc. 53 (1986), 237-266.

KP95 A. Kouvidakis and T. Pantev, The automorphism group of the moduli space of semi stable vector bundles, Math. Ann. 302 (1995), 225-268.

Lau88 G. Laumon, Un analogue global du cône nilpotent, Duke Math. J. 57 (1988), 647-671.

NN81 M. S. Narasimhan and M. V. Nori, Polarisations on an abelian variety, Proc. Ind. Acad. Sci. (Math. Sci.) 90 (1981), 125-128.

NR75 M. S. Narasimhan and S. Ramanan, Deformations of the moduli space of vector bundles over an algebraic curve, Ann. Math. 101 (1975), 391-417.

Sim92 C. T. Simpson, Higgs bundles and local systems, Inst. Hautes Études Sci. Publ. Math. 75 (1992), $5-95$.

Sim94a C. T. Simpson, Moduli of representations of the fundamental group of a smooth projective variety. I, Inst. Hautes Études Sci. Publ. Math. 79 (1994), 47-129.

Sim94b C. T. Simpson, Moduli of representations of the fundamental group of a smooth projective variety. II, Inst. Hautes Études Sci. Publ. Math. 80 (1994), 5-79.

ZT86 P. G. Zograf and L. A. Takhtadzhyan, Narasimhan-Seshadri connection and Kähler structure of the space of moduli of holomorphic vector bundles over a Riemann surface, Funct. Anal. Appl. 20 (1986), 240-241.

Indranil Biswas indranil@math.tifr.res.in

School of Mathematics, Tata Institute of Fundamental Research, Homi Bhabha Road, Bombay 400005, India 